\title{
Visual perceptive simultaneity and masking of letters successively presented
}

PAUL FRAISSE

SORBONNE, UNIVERSITY OF PARIS

Two sets of letters $S_{1}$ and $S_{2}$ when presented successively are perceived as simultaneous if the total duration of time from the beginning of $S_{1}$ to the end of $S_{2}$ is kept constant, whatever the duration of $S_{1}, S_{2}$, or the interval. The same law applies in the case of dots arranged to form geometrical figures. On the other hand, the phenomena of meta-contrast with letters are modified when the relative duration of $S_{1}$, $S_{2}$, and the interval vary. Thus perceptive integration and masking depend upon different processes.

Under what temporal conditions are letters successively presented seen as simultaneous or successive? This problem has not received much attention. Using four dots or lights arranged in such a way as to form a diamond, Lichtenstein (1961) found that, when flashed successively, the four dots were perceived as forming the diamond if the total duration between the lighting of the first and fourth dot did not exceed 125 msec., variations in the temporal intervals having no effect within these limits. Results are not so precise when letters or words are used as stimuli; however, Hylan (1903) had noticed that six letters are viewed as simultaneous, whatever the order of presentation maybe, provided the total duration does not exceed $80 \mathrm{msec}$. Stein (1928) showed that the letters of a word when flashed successively, in direct or in reverse order, were perceived in the same way as if the letters were presented simul- taneously, provided the total duration of successive flashes did not exceed $100 \mathrm{msec}$.

The purpose of this work is to verify and render more precise these results, and particularly: (a) to compare the laws of this phenomenon with letters or dots in a geometrical pattern; (b) to compare the laws of perceptive integration to those of lateral masking (or metacontrast) between letters themselves, when we change the duration of the stimuli and of the interval.

\section{Method}

With a three channel tachistoscope (Scientific Prototype model) the subject is presented with a preliminary stimulus (followed by a light interval of the same luminance) after which a second follows.

Six-letter words of which letters 1-3-5 form a word and letters 2-4-6 another, were chosen. Example: F L E U R I formed with FER and L U I. The first and the second words are respectively the first and the second stimuli. Letters are presented in such a way that in simultaneous presentation both stimuli are perceived as forming only one word. Capital letters are used (letters were $4 \mathrm{~mm}$ high and 3 to $4 \mathrm{~mm}$ thick). We used eight words of the same type as F L E U R I. Each subject was presented with two different words in order to neutralize the structural and frequency effects of the words.

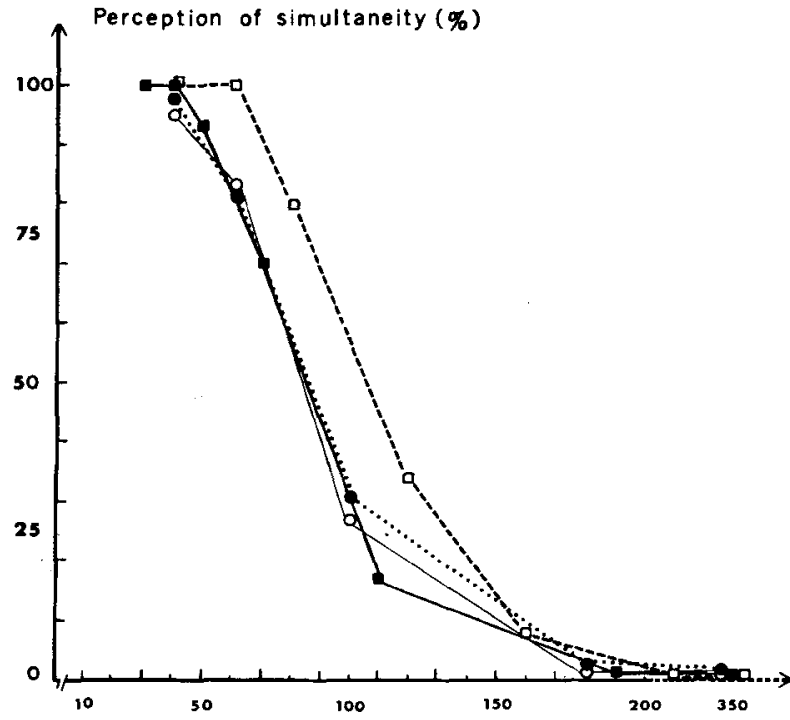

Fig.1a: LETTERS

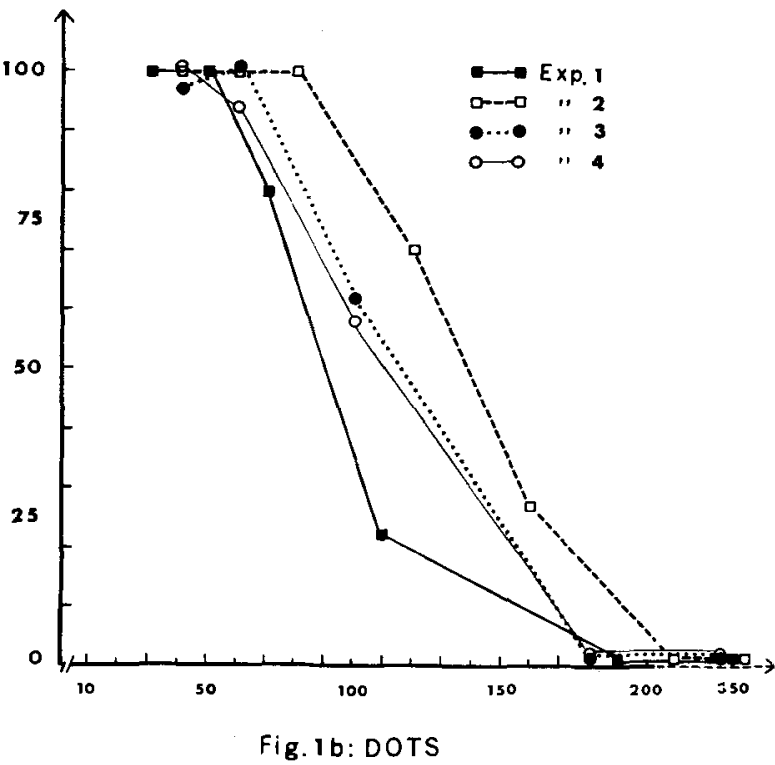

Fig. 1a and 1b. Percentage of perception of simultaneity according to the total duration (from the beginning of $S_{1}$, to the end of $S_{2}$ ) 
For figurative material we used black dots in a diamond pattern divided into two stimuli $\left(S_{1}\right.$ : two vertical dots; $\mathrm{S}_{2}$ : two horizontal dots) and in an hexagon pattern divided into two triangles of 3 points each.

The durations of $S_{1}, S_{2}$, and the interval determined four experimental situations:

Experiment 1: $\mathrm{S}_{1}=\mathrm{S}_{2}=15$ msec., with a varying interval from 0 to $320 \mathrm{msec}$.

Experiment 2: $S_{1}=S_{2}$, varying simultaneously from 20 to 180 msec.; interval $=0$

Experiment 3: $\mathrm{S}_{1}$ varies from 20 to $320 \mathrm{msec}$.; $\mathrm{S}_{2}=20 \mathrm{msec}$; interval $=0$

Experiment 4: $S_{1}=20$ msec.; $S_{2}$ varies from 20 to 320 msec.; interval $=0$

In each situation there were eight different subjects (male and female students). The presentation order (figures or words) was counterbalanced in each situation. For four subjects the variable duration was presented first in increasing order and then in decreasing order; the inverse order was used for the other four. Words and figures were presented twice at each step of duration.

As a result of our experimental design (eight subjects by two orders-increasing and decreasing-by two presentations), we obtained 64 values for letters and figures.

The subject was asked to say: (a) whether or not he perceived the stimuli successively; (b) the letters viewed in each stimulus.

\section{Resulis}

We have classified the responses of the subjects according to two categories: simultaneous perception or successive perception, even in the case of the content of the stimuli being partly perceived, or the case of interference (letters from the second stimulus being given as ones of the first or inversely).

The results show that experimental situations are
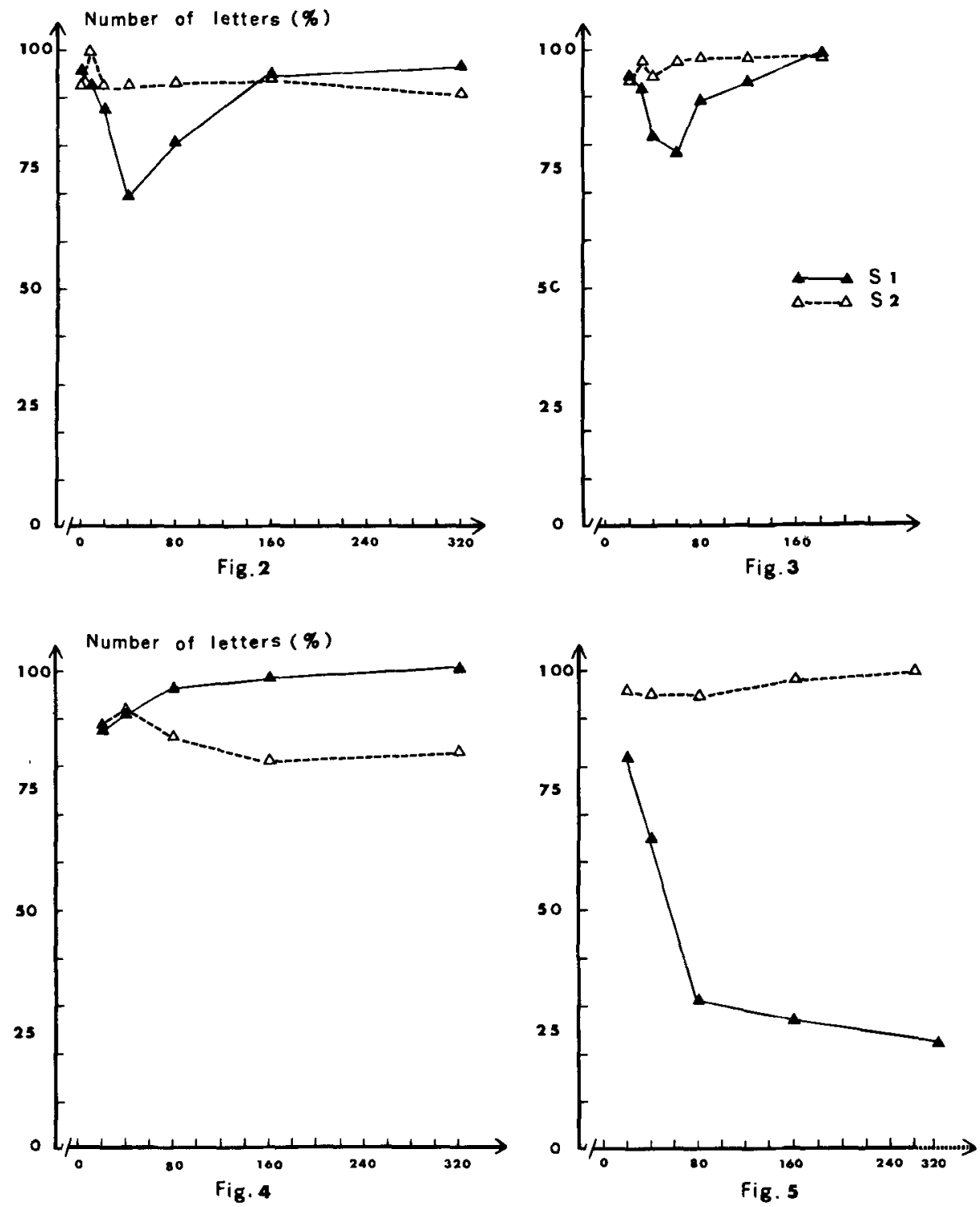

Fig. 2, 3, 4, 5. Percentage of recognized letters according to the variation of the duration of the interval (Fig. 2 , of $S_{1}, S_{2}$ (Fig. 3), of $s_{1}$ (Fig. 4), of $s_{2}$ (Fig. 5). 
comparable only if the total duration of the stimulation, from the beginning of $s_{1}$ to the end of $s_{2}$, is taken into account. The longer the duration, the more often there is perception of succession (Fig. 1a).

The phenomenon develops in the same way, whether or not there is an interval between the stimuli and whether or not the first stimulus is shorter, equal or longer than the second. It appears, however, that the situation where both stimuli have the same length, without an interval, is more favorable than the others. Results with dots are the same (Fig. 1b). This is true provided the duration of exposure of $s_{1}$ or $s_{2}$ is sufficient (here from 15 to $20 \mathrm{msec}$.). We must add that individual differences are important though for any subject numerical values obtained with letters and dots are almost the same. It can be concluded that in the case of durations less than $80-120$ msec. simultaneity rather than succession will be perceived.

In order to study the masking effect we have taken the number of perceived letters belonging to the first or the second stimulus, irrespective of the order in which they were given by the subjects, whether forming a word or isolated, and whether or not perceptive simultaneity occurred.

The rate of perceived letters related to the total duration of presentation $\mathrm{S}_{1}+\mathrm{i}+\mathrm{S}_{2}$ is given in Fig. 2, $3,4,5$. Lateral masking effects are closely linked to the particular situations. In Experiment 1 (Fig. 2) there is a maximum backward masking for a total duration of $70 \mathrm{msec}$., i.e., for a duration of $55 \mathrm{msec}$. between the beginning of $S_{1}$ and the beginning of $S_{2}$. In Experiment 2 (Fig. 3) the masking has the same nature with a maximum for a duration of $60 \mathrm{msec}$. between the beginning of $S_{1}$ and the beginning of $S_{2}$. In Experiment 3 (Fig. 4), $\mathrm{S}_{1}$ increases and there is a forward masking which increases with the duration of $S_{1}$. In Experiment 4 there is a backward masking which increases with the increase of duration of $\mathrm{S}_{2}$.

\section{Discussion}

Visual perceptive simultaneity appears within the same temporal limits, be the stimuli letters or geometric figures. Results are not affected by the meaning of the stimuli. On the other hand, the relative duration of the stimuli and the interval play an almost insignificant role.

These results confirm those obtained by the abovementioned authors and lend fresh support to the theses of those who believe in the existence of a psychological moment (Stroud, 1955; Lichtenstein, 1961; White, 1963).

However, specific modes of masking of the letters correspond to the diverse temporal conditions of the stimulation. We confirmed (a) the curve in U reported by Kolers (1962) and J. Blanc-Garin (1965), Weinstein and Haber (1965) and (b) an increasing effect of the masking when $\mathrm{S}_{2}$ increased, $\mathrm{S}_{1}$ being constant and an increasing forward masking when $s_{1}$ increased, $s_{2}$ being constant.

These phenomena of masking cannot be explained as a result of the processes which determine perceptive simultaneity, even if they take place in the same scale of duration.

\section{References}

Blanc-Garin, J. Quelques problemes posés par l'êtude du métacontraste visuel. Psychol. Franc., 1965, 65, 147-154.

Blanc-Garin, J. Les relations temporelles dans le masquage latéral visuel. Année Psychol., 1966, 66/2, in press.

Hylan, J. P. The distribution of attention. I. Psychol. Rev., 1903, 10, 373-403.

Kolers, P. A. Intensity and contour effects in visual masking. Vis. Res., 1962, 2, 277-294.

Lichtenstein, M. Phenomenal simultaneity with irregular timing of components of the visual stimulus. Percept. mot. Skills, 1961, 12, 47-60.

Stein, W. Tachistoskopisch Untersuchungen über das Lesen. Archiv. ges. Psychol., 1928, 64, 301-346.

Stroud, J. M. The fine structure of psychological time. In $\mathbf{H}$ Quastler (Ed.), Information theory in psychology. Glencoe, Ill. The Free Press, 1955. Pp. 174-207.

Weinstein, M., \& Haber, R. N. A U-shaped backward masking function in vision. Psychon. Sci., 1965, 2, 75-76.

White, C. T. Temporal numerosity and the psychological unit of duration. Psychol. Monogr., 1963, No. 575. 